\title{
EFEITOS DA MORTALIDADE GERAL BRASILEIRA SOBRE O CÁLCULO ATUARIAL: UMA COMPARAÇÃO ENTRE MODELOS PREDITIVOS ${ }^{1}$
}

\section{BRAZILIAN GENERAL MORTALITY EFFECTS ON ACTUARIAL CALCULATION: A COMPARI- SON BETWEEN PREDICTIVE MODELS}

\author{
Luiz Carlos Santos Júnior ${ }^{1}$ \\ Universidade Federal da Paraíba \\ luiz.atuario@gmail.com \\ Felipe Inácio Xavier de Azevedo \\ Universidade Estadual de Campinas \\ azevedofixa@hotmail.com \\ Miriam Harumi Tsunemi \\ Universidade Estadual Paulista \\ miriam.tsunemi@unesp.br
}

\section{RESUMO}

Objetivo: Objetiva-se neste trabalho analisar o efeito da mortalidade geral brasileira sobre o custo normal e a reserva matemática.

Fundamento: A necessidade de desenvolver modelos surge em um contexto de grandes mudanças nos padrões de mortalidade e de composição populacional, onde os modelos clássicos muitas vezes já não detêm o mesmo poder preditivo, em geral pela constância dos pressupostos assumidos para seu uso (Oliveira et al., 2012). No que tange ao comportamento da mortalidade em tempos recentes, os níveis decaíram para patamares muito além dos previstos a décadas atrás (Myrrha et al., 2014). A inconstância do evento passou a ser visado como um problema para a produção de estimativas fidedignas, atribuído às flutuações aleatórias, onde tanto os óbitos, quanto a população, ambos componentes da taxa de mortalidade, possuem influência nessas estimativas (Souza, 2014).

Método: Utilizam-se a Escala AA, o Modelo de Lee-Carter Estendido e o Modelo Quadrático Múltiplo, a partir de dados disponibilizados pelas tábuas do IBGE (2011-2016), além de dados de um Regime Próprio de Previdência Social (RPPS), fictício.

Resultados: Dentre os principais resultados, tem-se que: para ambos os sexos, a probabilidade de morte por idade se reduziu anualmente entre 2011 e 2016; para ambos os sexos, houve efeito da idade e de período sobre a mortalidade, mas não houve efeito de corte; houve ganho de longevidade para ambos os sexos; a mortalidade da mulher, em relação ao homem, é menor; a redução da mortalidade do homem, em relação

\footnotetext{
${ }^{1}$ Artigo recebido em: 23/10/2018. Revisado por pares em: 14/01/2019. Reformulado em: 21/02/2019. Recomendado para publicação em: 21/02/2019 por Anna Paola Freire (Editora Adjunta). Publicado em: 26/05/2019. Organização responsável pelo periódico: UFPB

${ }^{2}$ Endereço: Campus I - Lot. Cidade Universitaria, PB, 58051-900.
} 
a mulher, e do idoso, em relação aos mais jovens, é maior; o custo normal e a reserva matemática decorrentes das Tábuas AA e LC, em relação à Tábua 2016 observada, foram subestimados, enquanto a decorrente da Tábua MQM foi superestimada.

Contribuições: Apesar do interesse, no presente estudo, em mensurar o impacto das diferentes estimativas de mortalidade sobre o cálculo atuarial, seus efeitos ecoam, na verdade, sobre diversas áreas: sob a perspectiva demográfica, a mortalidade se constitui num dos três componentes (junto com a fecundidade e migração) que determinam o ritmo do crescimento populacional; sob o olhar das políticas públicas, em que a redução da mortalidade em alguma ou em todas as faixas etárias devem nortear a oferta de serviços públicos; sob a ótica previdenciária, define o tipo de relação entre ativos e inativos. Assim, de forma menos restrita, é possível compreender a importância de estudos relativos à mortalidade e suas inúmeras aplicações.

\section{Palavras-chave:}

Tábuas de vida do IBGE. Extrapolação e Projeção da Mortalidade. Expectativa de Vida. Cálculo Atuarial.

\section{ABSTRACT}

Objective: The objective of this study was to analyze the effects of brazilian general mortality on normal cost and mathematical reserve.

Background: The need for new standards to emerge in a context of major changes in mortality and population composition patterns is the fact that models often fail to detect the problem, in general, the constancy of the assumptions assumed for their use (Oliveira et al., 2012). Regarding the behavior of recent levels, levels have fallen to stages far beyond what was planned decades ago (Myrrha et al., 2014). The inconstancy of the event came to be seen as a problem of data production, attributed to random fluctuations, to one of the two components, relative to the population, to two components of the mortality rate, to a deviation in them (Souza, 2014).

Method: AA Scale, Extended Lee-Carter Model and Multiple Quadratic Model are used, based on data provided by the IBGE tables (2011-2016), as well as data from a fictitious Municipal Social Security System (RPPS).

Results: Among the main results, it has been observed that: for both sexes, the probability of death by age decreased annually between 2011 and 2016; for both sexes, there was age and period effect on mortality, but there was no cohort effect; there was a gain in longevity for both sexes; the mortality of women (in relation to men) is lower; the reduction in the mortality of men (in relation to women) and the elderly (in relation to the young) is higher; the normal cost and the mathematical reserve resulting from the AA scale and LC Tables, in relation to the 2016 observed Table, were underestimated, while that resulting from the MQM Table was overestimated.

Contributions: Despite the interest in the present study to measure the impact of different mortality estimates on the actuarial calculation, its effects do indeed resonate in several areas: from the demographic perspective, mortality is one of the three components (along with fecundity and migration) that determine the rate of population growth; under the eyes of public policies, in which the reduction of mortality in some or all age groups should guide the provision of public services; under the social security perspective, defines the type of relationship between assets and inactive assets. Thus, in a less restricted way, it is possible to understand the importance of studies on mortality and its many applications.

Keywords: IBGE life tables. Extrapolation and Mortality Projection. Life expectancy. Actuarial Calculation. 


\section{INTRODUÇÃO}

Ao longo do tempo, tem-se observado um aumento contínuo na expectativa de vida em praticamente todo o mundo, informação que evidencia a importância de mortalidade, suas causas e efeitos em diversos segmentos, quais sejam, atuarial, demográfico ou gestão pública, por exemplo.

O ganho em sobrevida constitui uma conquista social de grande importância para a sociedade, reflexo da queda sustentada da mortalidade em todas as idades, mas principalmente na infância (Camarano, \& Kanso, 2009). Essa conquista traz consigo novos desafios com implicações relevantes focadas no contexto de saúde e previdência. A queda da mortalidade não somente trouxe longevidade à população, mas decorrente do processo de transição demográfica, onde há uma queda combinada com os níveis de fecundidade, ocasionou um envelhecimento populacional, ou um maior contingente de pessoas com idades acima dos 60 anos (Miranda et al., 2016).

Cada vez mais pessoas alcançam idades avançadas e com isso há uma maior incerteza quanto aos níveis da mortalidade nessas idades. Turra (2012) destaca que o ganho de longevidade em idades avançadas tem se dado quase de forma linear nos últimos tempos, em especial para as mulheres. Apesar de haver um limite biológico, os avanços em tratamentos de doenças têm permitindo um maior tempo de vida e uma maior postergação da mortalidade, e assim, uma imprecisão maior das probabilidades de morte nessas idades.

Segundo Silva (2010), no contexto atuarial a preocupação se encontra na incerteza relativa ao evento morte, risco de longevidade, que define o tempo de percepção de benefícios e, por conseguinte, a Reserva Matemática (RM) - montante calculado atuarialmente, em determinada data, que expressa, em valor presente, o total dos recursos necessários ao pagamento dos compromissos do plano de benefícios ao longo do tempo (Brasil, 2008) - e o Custo Normal (CN) - valor pago ao Plano pelo participante ou seu mantenedor, (...), capaz de garantir os benefícios prometidos para a idade de elegibilidade (Rodrigues, 2008).

A modelagem da mortalidade, fator determinante no cálculo atuarial, é definida pela abordagem metodológica adotada (Wilbert et al., 2013). Nesse ínterim, existem diversas metodologias quando se trata de prever ou estimar a mortalidade - apesar de muitas técnicas terem se consolidado no meio atuarial, outras baseadas nas modelagens convencionais vêm sendo apresentadas como alternativas (Beltrão, \& Sugahara, 2002; Oliveira et al., 2012; Beltrão, \& Sugahara, 2017; Azevedo, 2018).

Assim, objetiva-se neste trabalho analisar a mortalidade geral brasileira e seus efeitos sobre o cálculo atuarial quando modificados os métodos de previsão, utilizando-se dos dados disponibilizados pelas tábuas masculina, feminina e ambos os sexos do IBGE, de 2011 a 2016, além de dados de um RPPS de pequeno porte fictício.

\section{FUNDAMENTAÇÃO TEÓRICA}

Grande parte dos RPPSs municipais adota como premissa biométrica a tábua do IBGE (esta tábua é o parâmetro mínimo a ser empregado nas avaliações atuariais, como determina a legislação por meio do § I do art. $6^{\circ}$ da Portaria № 403/2008), o que justifica a sua escolha. Ela fornece, no entanto, informações sobre a mortalidade para pessoas entre 0 e 80 anos de idade, sendo necessário extrapolar tal informação para além dessa idade, em conformidade com as idades alcançadas pelos participantes dos Institutos de Previdência. Deste modo, os termos "extrapolação" e "extrapolar" possuem, no presente trabalho, o significado de "obter valores de mortalidade que ultrapassem a idade de 80 anos". Para ilustrar, citam-se os trabalhos de Castro (2018), Namboodiri(1991) e ElandtJonshon e Johnson (1980), que propuseram modelos de extrapolação da mortalidade. 
Assim, a partir de uma série histórica de tábuas já extrapoladas, é possível, em seguida, projetar a mortalidade para fins de cálculos atuariais. Para sua realização, o ideal é que a tábua de vida possua boa amplitude, ou seja, possua última idade de aproximadamente 110 anos (Castro, 2018).

Para projetar a mortalidade, opta-se pelo uso de métodos que assentam na predição baseada nas tendências históricas registradas na mortalidade (Bravo, 2007), sendo o método de Lee-Carter (LC) um dos mais utilizados. Lee e Carter (1992) apresentaram um método para a modelagem e previsão das taxas de mortalidade específicas por idade e avaliaram a mortalidade norte-americana.

A partir daí, diversos trabalhos aplicaram e/ou estenderam seu modelo: Santos (2007) utilizou as metodologias LC e modelos lineares generalizados para construir previsões de mortalidade com base na experiência da população da Inglaterra e País de Gales da última metade do século passado; Gallop (2008) discutiu sobre motivos que podem modificar / influenciar a mortalidade no Reino Unido no século XXI, além de discutir a metodologia e as premissas usadas nas últimas projeções; Li, Lee e Gerland (2013) usaram dados do Japão e EUA para analisar a mortalidade em idades mais jovens e mais avançadas, por meio do método de Lee-Carter estendido; Huang e Browne (2017) projetaram taxas futuras de mortalidade para uso atuarial com dados chineses, por meio de uma modificação do Modelo de Investigação de Mortalidade Contínua.

Muitos outros modelos foram sendo desenvolvidos a partir dos modelos clássicos como o de LC, com inúmeras abordagens, modelagens, dados e aplicações diversas. No entanto, sua maioria se preocupa em produzir estimativas representativas, sejam em relação as suas populações, mas principalmente em relação às novas tendências da mortalidade (Beltrão, \& Sugahara, 2017).

A necessidade de desenvolver modelos surge em um contexto de grandes mudanças nos padrões de mortalidade e de composição populacional, onde os modelos clássicos muitas vezes já não detêm o mesmo poder preditivo, em geral pela constância dos pressupostos assumidos para seu uso (Oliveira et al., 2012). No que tange ao comportamento da mortalidade em tempos recentes, os níveis decaíram para patamares muito além dos previstos a décadas atrás (Myrrha et al., 2014). A inconstância do evento passou a ser visado como um problema para a produção de estimativas fidedignas, atribuído às flutuações aleatórias, onde tanto os óbitos quanto a população, ambos componentes da taxa de mortalidade, possuem influência nessas estimativas (Souza, 2014).

Não somente a redução da mortalidade e o ganho na esperança de vida, mas a mudança na estrutura populacional também implicou em necessidades específicas em relação às estimativas de mortalidade. A divisão das tábuas de vida por idade e sexo ainda retratam diferenciais importantes no que tange às políticas públicas, mas novas demandas são apontadas. As estimativas por níveis de escolaridade e renda, a decomposição das estimativas de morte por causas, são algumas das abordagens recentes com foco previdenciário e de saúde (Ribeiro et al., 2016).

Além desses fatores, os níveis e recortes populacionais ou espaciais requerem modelos mais robustos que captem o comportamento da mortalidade em pequenas escalas ou contextos desagregados, onde os modelos clássicos não se aplicam com a mesma qualidade como para grandes áreas (Gonzaga, \& Schmertmann, 2016).

No contexto de pequenas áreas, no nível municipal, por exemplo, onde a maioria dos RPPSs se enquadra, a variabilidade da mortalidade é mais intensa e requer modelos mais robustos para previsão dessas estimativas. O comportamento da mortalidade pode diferir do contexto macro por diversos motivos que englobam condições sociais e de desenvolvimento socioeconômico local, mas também pelo volume populacional (Souza, 2014; Gonzaga, \& Schmertmann, 2016). A ausência de estimativas nesse nível leva ao uso de estimativas médias que muitas vezes não são tão aderentes 
ou representativas de suas populações de beneficiários (Wilbert et al., 2013). Nesse sentido, a proposição de novos modelos tem contribuído para, se não preencher, amenizar essa lacuna.

Atreladas às estimativas de mortalidade, as tábuas de vidas são ferramentas importantes e quanto mais desagregadas e aderentes às populações, melhores as previsões e estimativas do cálculo atuarial. A desagregação das idades, de grupos etários, para idades simples, proporciona uma estimativa pontual melhor aproximada ao nível individual como requerido para os cálculos previdenciários, por exemplo. Em geral a desagregação pode se dar por meio de regressão ou processos de interpolação. Alguns métodos que propõem a desagregação da mortalidade por idades simples embutem processos de suavização que muitas vezes distorcem as estimativas (Judson, \& Popoff, 2004).

Depois de resumida a importância dos estudos acerca da mortalidade, tem-se que o presente trabalho se propõe a investigar o seu comportamento e suas previsões (decorrentes de três modelos). De antemão, informa-se que houve ganho de longevidade e que o tempo médio restante de vida aumentará para todas as idades, segundo as projeções apresentadas na seção de Resultados. Além disso, não existem evidências para rejeitar a hipótese nula de que as distribuições das tábuas aqui estimadas se diferem da distribuição da tábua-referência. Consequência disso é que para os dados simulados, as variações percentuais de CN e RM são pequenas quando comparadas ao cenário-referência.

\section{PROCEDIMENTOS METODOLÓGICOS}

Esta seção apresenta as informações analisadas, os modelos de extrapolação e previsão da mortalidade e as formulações para cálculo atuarial. Deste modo, a ordem de procedimentos necessária para a realização da pesquisa é: conforme as características dos dados (subseção 3.1), opta-se por extrapolar a informação referente à mortalidade (subseção 3.2), para em seguida predizê-la, conforme os modelos conhecidos como "Escala AA" (subseção 3.3.1), Lee-Carter estendido (subseção 3.3.2) e MQM (subseção 3.3.3). Por fim, as três estimativas são testadas (subseção 3.4) e aplicadas nas formulações atuariais, para cálculo da RM e CN, segundo fixação de premissas e dados simulados (subseção 3.5).

\subsection{Apresentação dos Dados}

Utilizam-se dados secundários, isto é, as tábuas do IBGE compreendidas entre 2011 e 2016, Masculinas e Femininas (discussão inicial) e ambos (aplicação nos cálculos atuariais) - disponíveis no site do IBGE - para obtenção da mortalidade extrapolada e prevista.

Depois de estimadas, as mortalidades (conforme cada modelo proposto) são aplicadas ao cálculo atuarial, segundo as premissas aqui estipuladas e em conformidade com os dados simulados de um RPPS fictício.

\subsection{Extrapolação de Mortalidade}

Castro (2018) apresentou um método de extrapolação da mortalidade para idades acima de 80 anos constituído por nove etapas. Tal método encontra um Fator de Ajuste (FA) a ser utilizado na formulação do número de sobreviventes a idade $\mathrm{x}\left(\mathrm{l}_{\mathrm{x}}\right)$, de modo que a esperança de sobrevida a partir dos 80 anos $\left(\mathrm{e}_{80}\right)$ seja igual àquela calculada pelo IBGE e disponibilizada nas tábuas.

1) Calcular o número de mortes $d_{x}$ relativos às idades de 0 a 79 anos, a partir da probabilidade de morte por idade $q_{x}$ disponibilizados pelo IBGE: 


$$
d_{x}=\frac{q_{x}}{1000} \times l_{x}
$$

2) A raiz da tábua é $l_{0}=100.000$. A partir dela, calcular os $l_{x}$ para $x=1, \ldots, 80$ anos:

3) Calcular $l_{x}$ por idade acima de 80 anos:

$$
l_{x+1}=l_{x}-d_{x} \text {. }
$$

$$
l_{x+2}=l_{x+1} \times\left(\frac{l_{x+1}}{l_{x}+F A}\right) .
$$

Iniciam-se os cálculos com FA igual a 100, calculando $\mathrm{l}_{\mathrm{x}}$ até $\mathrm{x}=115$ anos $\left(\mathrm{l}_{115}\right)$.

4) A partir dos $l_{x}$ encontrados até $x=115$ anos, calcular $d_{x}$ para $x=80, \ldots, 115$ anos:

$$
d_{x}=l_{x}-l_{x+1} \text {. }
$$

5) A partir de $l_{x}$ e $d_{x}$ encontrados até $x=115$ anos, calcular os $q_{x}$ para $x=80, \ldots, 115$ anos:

$$
q_{x}=\frac{d_{x}}{l_{x}}
$$

6) A partir dos $l_{x}$ encontrados, calcular $L_{x}$, isto é, o número de anos vividos pela geração $l_{0}$ entre as idades $\mathrm{x}$ e $\mathrm{x}+\mathrm{n}$, para $\mathrm{x}=0, \ldots, 115$ anos, utilizando-se a fórmula do fator de separação das mortes $\left(f_{x}\right)$ :

$$
L_{x}=f_{x} \times l_{x}+\left(1-f_{x}\right) \times l_{x+1}
$$

Na primeira idade $\left(f_{0}\right), f_{x}$ assume valores entre 0,05 e 0,35 ; para as outras idades, assume valor igual a 0,5 , pois as mortes acabam ocorrendo de forma uniforme ao longo do ano, em média, na metade dele (CASTRO, 2018). Como no primeiro ano de vida as mortes não se distribuem uniformemente ao longo do ano, calcular $\mathrm{f}_{0}$ da tábua do IBGE:

$$
f_{0}=\frac{\left(L_{0}-l_{1}\right)}{d_{0}}
$$

com $\mathrm{d}_{0}$ e $\mathrm{l}_{1}$ calculados nas etapas 1 e 2 .

7) A partir dos $L_{x}$ encontrados, calcular $T_{x}$, ou seja, o número total de anos vividos pela geração $l_{0}$ entre as idades $x$ e $\omega$ (última idade da tábua), para $x=0, \ldots, 115$ anos:

$$
T_{x}=\sum_{a=x}^{\omega-1} L_{a}=L_{x}+L_{x+1}+\ldots+L_{\omega-1} .
$$

8) A partir dos $T_{x}$ e $l_{x}$ encontrados, calcular $e_{x}$ (esperança de sobrevida na idade $x$ ) para $x=$ $0, \ldots, 115$ anos:

$$
e_{x}=\frac{T_{x}}{l_{x}}
$$

9) Depois de calculadas as esperanças de sobrevida na idade $x$, observa-se o valor da esperança de sobrevida na idade de 80 anos $\left(\mathrm{e}_{80}\right)$ resultante da utilização de $F A=100$. $O$ passo seguinte consiste em modificar o valor de FA até que $\mathrm{e}_{80}$ calculada convirja para $\mathrm{e}_{80}$ divulgada pelo IBGE. Para mais detalhes sobre o método, consultar Castro (2018). 


\subsection{Previsão de Mortalidade}

É sabido que o Brasil vem observando um aumento contínuo na expectativa de sobrevida de seus habitantes. Em decorrência disso, os atuários são conduzidos a rever, periodicamente, as tábuas de mortalidade adotadas como hipótese atuarial, como uma medida prudencial para minimizar o risco de longevidade. Nesse contexto, as tábuas geracionais surgiram como uma forma de incluir nos modelos atuariais um acompanhamento das oscilações demográficas, já que contemplam aumentos na expectativa de vida dos participantes de um plano através de um contínuo decréscimo da probabilidade de morte a cada período (Nunes, \&Carvalho, 2008).

Como a mortalidade não permanece constante em relação ao tempo, introduz-se a discussão acerca dos Fatores de Improvement (FI), isto é, fatores de redução de mortalidade que consideram, durante a construção de tábuas de mortalidade, a mudança dos padrões de mortalidade ao longo dos anos. Eles consideram dependência entre as covariáveis e o tempo, incorporando o ganho de longevidade da população.

\subsection{Escala AA}

Os métodos utilizados para estimar o improvement envolvem o uso de modelos complexos e de séries históricas de mortalidade com determinado grau de qualidade, o que faz com que se opte por modelos alternativos. Visando minimizar tal problema, algumas tábuas fazem uso do método de improvement por escala de projeção, que traz fatores (distribuídos por idade e sexo) de melhoria anual da mortalidade. Por exemplo, comitês da Society of Actuaries criaram a Escala AA (Anual Improvement Factor) para projeções de mortalidade a partir de suas tábuas básicas (Nunes, \& Carvalho, 2008).

A escala AA, de uso comum nos Estados Unidos, foi construída baseada nas experiências do Federal Civil Service e do Social Security norte-americanos, no período de 1977 a 1993, com os seguintes ajustes em adição à suavização das tendências de mortalidade: uma tendência de improvement mínima de $0,5 \%$ ao ano antes da idade 85 ; uma tendência de improvement máxima de $2 \%$ ao ano; tendências classificadas em 0,1\% à idade 100 (Santos, 2007).

Conforme notação de Nunes e Carvalho (2008), a projeção de mortalidade a partir da escala de projeção AA é dada por:

$$
q_{\text {projetado }}=q_{x} \times\left(1-s_{x}\right)^{n}
$$

em que $\mathrm{q}_{\mathrm{x}}$ é a probabilidade de morte de um indivíduo com idade $\mathrm{x}$ segundo umatábua básica qualquer; $s_{\mathrm{x}}$ é o fator de melhoria anual para a idade $\mathrm{x}$ trazido pela escala utilizada; en é o número de períodos (em anos) que se deseja projetar a probabilidade de referência.

É possível notar, por meio da visualização da equação (1), que as melhorias sofridas pelas taxas são lineares, ou seja, o método assume que a longevidade aumenta linearmente com o passar do tempo.

Segundo Nunes e Carvalho (2008), a tábua projetada pela escala, apesar de ajustar suas taxas, não incorpora uma melhoria continuada das expectativas de vida. Isso significa que suas taxas se tornam defasadas à medida que o ano-calendário se distanciado ano base da tábua. Como uma alternativa a esse problema, foram criadas as tábuas geracionais, tema abordado na subseção 3.3.2. 


\subsection{Modelo de Lee-Carter Estendido}

De acordo com a revisão de literatura, a projeção de ganho ou perda de longevidade se dá principalmente por meio do método de Lee-Carter ou por meio de Modelos Lineares Generalizados (MLG).

Segundo Booth e Tickle (2008), os métodos que usam modelos de dois fatores (idade-período ou idade-coorte) são mais bem-sucedidos. O método Lee-Carter de dois fatores e, em particular, suas variantes, obtém sucesso em termos de precisão, enquanto os avanços recentes melhoraram a estimativa da incerteza prevista. Métodos baseados em regressão (MLG) têm tido menos sucesso, devido a não linearidade no tempo.

O método de Lee-Carter (LC) é dado por um modelo de estimação de uma matriz de taxas específicas de mortalidade no tempo t para a idade $\mathrm{x}\left(\mathrm{m}_{\mathrm{x}}\right)$ através da estimativa de mínimos quadrados como solução para a equação:

$$
\ln \left(m_{x, t}\right)=\alpha_{x}+\beta_{x} k_{t}+\varepsilon_{x, t}
$$

onde $\alpha_{\mathrm{x}}$ representa um padrão constante de mortalidade por idade; $\beta_{\mathrm{x}}$ descrevem a tendência da mortalidade à idade $\mathrm{x}$ quando o nível geral de mortalidade $\mathrm{k}_{\mathrm{t}}$ se altera; $\mathrm{k}_{\mathrm{t}}$ é a tendência da mortalidade ao longo do tempo; $\varepsilon_{\mathrm{x}, \mathrm{t}}$ é um termo de erro (efeitos aleatórios) com distribuição normal de média zero e variância constante $\sigma^{2}$. Os detalhes do processo de estimação dos parâmetros $\alpha_{\mathrm{x}}, \beta_{\mathrm{x}} \mathrm{e}$ $\mathrm{k}_{\mathrm{t}}$ também são apresentados por Monteiro e Leão (2012) e Butt, Haberman e Shang (2014).

Um ponto positivo do método é o fato de combinar um modelo demográfico com um modelo de séries temporais, permitindo obter intervalos probabilísticos para as respectivas previsões. O método também permite que as taxas de mortalidade decresçam de forma exponencial sem o estabelecimento de um limite arbitrário (Monteiro, \&Leão, 2012).

Em um desenvolvimento mais recente, a configuração básica foi ampliada para incluir um termo adicional, contendo um efeito de segundo período ou um efeito de coorte, como em Renshaw e Haberman (2003). O modelo LC básico pode ser transformado num quadro mais geral para analisar a relação entre idade e tempo e seu impacto conjunto sobre as forças de mortalidade (Butt, Haberman, \& Shang, 2014).

$$
\mu_{x, t}=\exp \left(\alpha_{x}+\beta_{x}^{0} l_{t-x}+\beta_{x}^{1} \times k_{t}\right)
$$

onde $\alpha_{\mathrm{x}}$ mapeia o perfil de idade principal da mortalidade; $\mathrm{l}_{\mathrm{t}-\mathrm{x}}$ e $\mathrm{k}_{\mathrm{t}}$ representam os efeitos de coorte e período, respectivamente; os parâmetros $\beta_{\mathrm{x}}^{0}$ e $\beta_{\mathrm{x}}^{1}$ medem as interações correspondentes com a idade.

Observa-se que o modelo (2) representa uma família de seis modelos não-lineares generalizados da estrutura do tipo LC com função de ligação logarítmica. As subcategorias do modelo geral são determinadas definindo-se os parâmetros de interação $\left(\beta_{\mathrm{x}}^{0,1}\right)$ : a) desconhecido (a ser estimado); = 1 (fixo); $=0$ (vazio). Assim, a estrutura do tipo LC básico resulta da definição dos parâmetros específicos da idade como: $\beta_{\mathrm{x}}^{0}=0(\forall \mathrm{x})$ e $\beta_{\mathrm{x}}^{1}=\beta_{\mathrm{x}}$.

Por fim, utilizam-se dos valores de $\mathrm{q}_{\mathrm{x}}$ estimados por LC para posterior cálculo daVida Média Residual (Vmr): para indivíduos com idade x esta quantidade mede o tempo médio restante de vida. Assim, segundo Colosimo e Giolo (2006) 


$$
\operatorname{Vmr}(x)=\frac{\int_{x}^{\infty} S(u) d u}{S(x)} \text {. }
$$

A operacionalização desta análise foi realizada no programa R, por meio do pacote ilc (Butt, Haberman, \& Shang, 2014) e códigos a parte.

\subsection{Modelo Quadrático Múltiplo}

A ideia do modelo se baseia em uma série de probabilidade de morte por idade ao longo do tempo, onde se aplica uma dupla regressão para obtenção do Modelo de Múltiplo Quadrático (MQM), uma vez que em ambas as regressões os modelos definidos são do tipo quadrático. Azevedo(2018) constrói o modelo com o uso das probabilidades de morte por idade simples obtidas da série histórica e projeções do IBGE. Ele propõe que as probabilidades sejam submetidas a um processo de alisamento exponencial e que os modelos sejam construídos a partir dos logaritmos das probabilidades de morte. Esse processo possibilita melhor ajuste do modelo por suavizar distorções da mortalidade ao longo da curva.

O comportamento da mortalidade a cada idade no tempo é definido por um modelo quadrático. Utilizando regressão por mínimos quadrados o autor obtém os parâmetros do modelo para cada idade. O modelo quadrático possui a seguinte estrutura:

$$
y_{x}^{t}=c_{x}+\beta_{x} t+\alpha_{x} t^{2}
$$

em que, $y_{x}^{t}$ representaa probabilidade de morte à idade $x$ no tempot. Por sua vez, $c_{x}$ é o coeficiente de ajuste do nível da curva de mortalidade, $\beta_{\mathrm{x}} \mathrm{e} \alpha_{\mathrm{x}}, \alpha \neq 0 \forall \mathrm{x}$, são os coeficientes de ajuste do deslocamento e direção da curva, respectivamente.

Para cada idade simples há um modelo quadrático que contém os parâmetros, $\mathrm{c}, \beta$ e $\alpha$. Dado que o comportamento desses parâmetros ao longo das idades é explicado também por um modelo quadrático, uma nova regressão é aplicada sobre esse conjunto de parâmetros, retornando três novos modelos quadráticos. Essa regressão pressupõe que a mortalidade entre idades, $\mathrm{x}$ e $\mathrm{x}+1$, está correlacionada. Desse modo, o modelo quadrático assume a seguinte equação:

$$
y_{x}^{t}=f_{x}(c)+f_{x}(\beta) \boldsymbol{t}+f_{x}(\alpha) \boldsymbol{t}^{2}
$$

Substituindo por funções quadráticas obtém-se o modelo múltiplo quadrático:

$$
y_{x}^{t}=\left(c+\beta \boldsymbol{x}+\alpha \boldsymbol{x}^{2}\right)+\left(c+\beta \boldsymbol{x}+\alpha \boldsymbol{x}^{2}\right) \boldsymbol{t}+\left(c+\beta \boldsymbol{x}+\alpha \boldsymbol{x}^{2}\right) \boldsymbol{t}^{2} .
$$

Para o autor, esse modelo congrega um modelo temporal com um modelo relacional etário, composto por duas componentes variáveis, a idade, $\mathrm{x}$, e o tempo, t, os demais sãoparâmetros fixos advindos dos modelos de regressão.

Uma vez de posse dos parâmetros é possível estimar a mortalidade para qualquer idade, dentro do rol de idades utilizadas para a construção do modelo, em qualquer momento no tempo. Vale destacar que considerando que o modelo foi construído com o uso das probabilidades de morte em escala logarítmica, as estimativas vão ser retomadas na mesma escala, o que se faz necessário aplicar a função inversa, o exponencial, para obtenção das probabilidades de morte. 


\subsection{Teste de Aderência e Medida de Acurácia}

Depois de estimada a mortalidade, é necessária a realização de um teste de aderência para investigar se as idades estimada e observada possuem a mesma distribuição probabilística. No meio atuarial é comum a utilização do teste Kolmogorov-Smirnov (KS).

Esse teste, para o caso aqui proposto, averigua a seguinte hipótese: para duas amostras, comprova se ambas foram extraídas da mesma população, e segundo Bravo (2007), pode ser usado para comparar as distribuições do número de óbitos esperados $\left(D_{o b s}=\sum_{k=x_{\min }}^{x_{\max }} d_{k}\right)$ e observa$\operatorname{dos}\left(D_{\text {esp }}=\sum_{\mathrm{k}=\mathrm{x}_{\min }}^{\mathrm{x}_{\max }} \mathrm{d}_{\mathrm{k}}^{\mathrm{esp}}\right)$ entre as idades mínima $\left(\mathrm{x}_{\min }\right)$ e máxima $\left(\mathrm{x}_{\max }\right)$. Considere, ainda, que:

$$
F(x)=\frac{1}{D_{o b s}}\left(\sum_{k=x_{\min }}^{\mathrm{x}} d_{k}\right) \text { e } G(x)=\frac{1}{D_{e s p}}\left(\sum_{k=x_{\min }}^{\mathrm{x}} d_{k}^{e s p}\right)
$$

em que $d_{k}$ é o número de óbitos ocorridos entre as idadesk e $k+1 ; d_{k}^{e s p}$ é o número de óbitos esperados entre as idadesk e $k+1$. Assim, a estatística do teste $\left(W^{K S}\right)$ é:

$$
W^{K S}=D e v^{\max }\left(\frac{D_{o b s} \times D_{e s p}}{D_{o b s}+D_{e s p}}\right)^{0,5} .
$$

$\operatorname{Dev}^{\max }=\operatorname{Max}_{\mathrm{x}}|\mathrm{F}(\mathrm{x})-\mathrm{M}(\mathrm{x})|$ é a diferença máxima entre as duas distribuições. $\mathrm{W}^{\mathrm{KS}}$ tem distribuição conhecida, e a partir dela é possível avaliar a rejeição ou não da hipótese nula, isto é, há a igualdade de distribuições entre o número de óbitos observados e esperados.

Segundo Khoury (2011), a Média Percentual Absoluta do Erro (MAPE) expressa a acurácia do erro em porcentagem,

$$
M A P E=\frac{\sum_{i=1}^{n}\left|\left(\left(y_{i}-\hat{y}_{i}\right) / y_{i}\right)\right|}{m} \times 100, \text { sey }_{i} \neq 0
$$

em que $y_{i}$ são os tempos observados; $\hat{y}_{i}$ são os tempos ajustados; $m$ é o número de observações no tempo $t$. Quando $y_{i}=\hat{y}_{i}$, obtém-se $M A P E=0$, ou seja, quanto menor o MAPE, melhor o ajuste.

Apesar de ser um teste recorrente na literatura, outras técnicas ainda mais robustas vêm sendo desenvolvidas no âmbito da modelagem para avaliar a qualidade de modelos e seus ajustes (Deprez et al. 2017; Sakr et al., 2017; Richman, 2018). A capacidade de processamento de dados com o uso de tecnologias modernas permite maior acurácia dos modelos. Nesse contexto, as técnicas de Machine Learning (ML) compõem essas abordagens robustas, e mais recentemente tem sido utilizada nas áreas de demografia e atuária (Richman, 2018).Este autor, por exemplo, apresenta uma vasta literatura exemplificando como o processo de aprendizado por meio do ML pode resultar num desfecho atuarial; e explora como as técnicas de ML podem ser aplicadas e incorporadas na atuária com aplicações na modelagem da mortalidade e precificação.

\subsection{Formulações para Cálculo Atuarial}

Em planos capitalizados de Benefício Definido (BD), a Reserva Matemática (RM)acumulada até a idade $\mathrm{x}$, formada a partir da acumulação e capitalização das contribuições (dos segurados e patronais), segue a formulação (Correa, 2018): 


$$
R M_{x}=B_{x} \times_{r-x} p_{x}^{T} \times v^{r-x} \times \ddot{a}_{r} .(6)
$$

$\mathrm{B}_{\mathrm{x}}$ é o valor de benefício acumulado entre as idades y e $\mathrm{x}$; $\ddot{\mathrm{a}}_{\mathrm{r}}$ corresponde à anuidade vitalícia a partir da idade $\mathrm{r} ; \quad \mathrm{r}-\mathrm{x} \mathrm{p}_{\mathrm{x}}^{\mathrm{T}}$ é a probabilidade de o indivíduo sobreviver por todas as causas entre as idades $\mathrm{x}$ e $\mathrm{r} ; \mathrm{v}^{\mathrm{r}-\mathrm{x}}$ é a taxa de desconto que traz o valor do benefício acumulado $\mathrm{B}$ a valor presente.

A concepção das formulações requer definições mais precisas e práticas acerca da Reserva Matemática. Apesar das distintas formas de se obter esses montantes, com variações metodológicas, no cerne, o pressuposto dessas medidas é o mesmo. Para Bowers et al, (1997, p.205) “[...] the benefit reserve at time tis the conditional expectation of the difference between the present value of future benefits and the present value of future benefit premiums, the conditioning event being survivorship of the insured to time $t^{\prime \prime}$.Essa definição explicita o risco de morte e da mudança do dinheiro no tempo: quando mal estimados, implica em mau dimensionamento da RM e consequente desequilíbrio do plano de benefícios (Pinheiro, 2007).

O valor da contribuição - Custo Normal $(\mathrm{CN})$ - é dado pela expressão:

$$
C N_{x}=b_{x} \times_{r-x} p_{x}^{T} \times v^{r-x} \times \ddot{a}_{r} .(7)
$$

Em que $\mathrm{b}_{\mathrm{x}}$ é o acúmulo anual (parcial ou marginal) do benefício total $\mathrm{B}_{\mathrm{r}}$, de modo que:

$$
B_{r}=\sum_{t=y}^{x-1} b_{t} \cdot(8)
$$

Em que y é a idade de início de contribuição; $r$ é a idade de concessão de aposentadoria; $\mathrm{B}_{\mathrm{r}}$ é o valor de benefício acumulado entras as idades y e r (durante seu período de atividade).

Existem diversas definições de $\mathrm{b}_{\mathrm{x}}$ - diversos métodos de custeio - e, consequentemente, diferentes valores de $\mathrm{RM}_{\mathrm{x}}$ e $\mathrm{CN}_{\mathrm{x}}$ calculados.Um método de custeio define as fontes de recursos necessárias para o financiamento dos benefícios oferecidos pelo Plano de Benefícios e taxa de administração, representadas pelas alíquotas de contribuições previdenciárias a serem pagas pelo ente federativo, pelos servidores ativos e inativos e pelos pensionistas ao respectivo RPPS e aportes necessários ao atingimento do equilíbrio financeiro e atuarial, com detalhamento do custo normal e suplementar (BRASIL, 2008).

No método Crédito Unitário Projetado (PUC), método aqui adotado- por ser, em conjunto com o método de Idade de Entrada Normal (IEN), o mais utilizado pelos RPPSs (Colatto, \& Dias, 2018) , o benefício adquirido no início do ano x é determinado pelo benefício projetado na aposentadoria $\left(B_{r}\right)$ e sua representação matemática é (Winklevoss, 1993):

$$
B_{x}=\frac{B_{r}}{(r-y)} \times(x-y) \cdot(9)
$$

Assim, o custo normal é igual ao valor presente dos benefícios projetados dividido pelos anos de serviço até a aposentadoria; os salários de contribuição também são projetados até a data de aposentadoria pelas taxas de crescimento salarial (s).Como o participante paga, a cada ano, um percentual fixo de seu salário, o peso da contribuição é constante no tempo, acompanhando seu padrão de consumo; a correta acumulação dos valores devidos depende da concretização das premissas sobrea variação do salário do participante. 


\section{RESULTADOS}

Conforme os objetivos e métodos já explanados, apresentam-se nesta seção os resultados referentes à extrapolação e previsão da mortalidade, bem como seu efeito sobre o cálculo atuarial.

\subsection{Extrapolação e Previsão da Mortalidade}

A partir das tábuas do IBGE (que possuem informações para indivíduos de até 80 anos) masculinas e femininas, de 2011 a 2016, e por meio do método de Castro (2018), extrapolaram-se as mortalidades para as idades superiores a 80 anos, conforme a Tabela 1.

Tabela 1 - Extrapolação das mortalidades (masculina e feminina)

\begin{tabular}{cccccccc}
\hline \multirow{2}{*}{ Idade } & \multicolumn{2}{c}{2011} & & $\ldots$ & & \multicolumn{2}{c}{2016} \\
\cline { 2 - 7 } & $q_{x}$ masc. & $q_{x}$ fem. & $\ldots$ & $\ldots$ & $q_{x}$ masc. & $q_{x}$ fem. \\
\hline 81 & 0,0784892 & 0,0590202 & $\ldots$ & $\ldots$ & 0,07252443 & 0,0525623 \\
$\vdots$ & $\vdots$ & $\vdots$ & $\ddots$ & $\ddots$ & $\vdots$ & $\vdots$ \\
105 & 0,999792 & 0,9814721 & $\ldots$ & $\ldots$ & 0,98126857 & 0,6189003 \\
106 & 1 & 0,9995979 & $\ldots$ & $\ldots$ & 0,99958867 & 0,7617988 \\
107 & 1 & 0,9999998 & $\ldots$ & $\ldots$ & 0,99999983 & 0,907464 \\
108 & 1 & 1 & $\ldots$ & $\ldots$ & 1,00000000 & 0,9878378 \\
109 & 1 & 1 & $\ldots$ & $\ldots$ & 1,00000000 & 0,999832 \\
110 & 1 & 1 & \multicolumn{2}{c}{ Fonte: Elaboração Própria (2018) }
\end{tabular}

As tábuas do IBGE de 2011 a 2016, agora extrapoladas (até a idade 110 anos), foram utilizadas como série histórica para projeção da mortalidade. As Figuras 1 (masculino) e 2 (feminino) apresentam o logaritmo da probabilidade de morte por idade por tábua.

Figura 1 - Log da probabilidade de morte por idade da tábua do IBGE (masculino), de 2011 a 2016

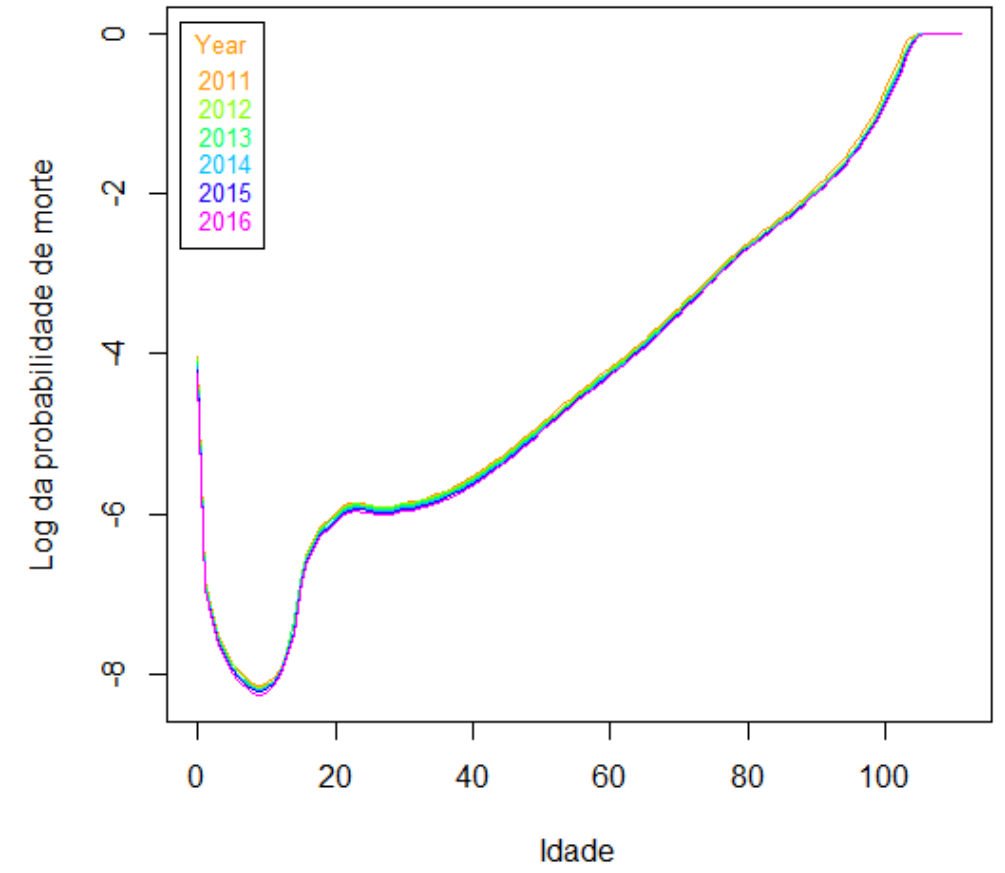

Fonte: Elaboração Própria (2018) 
Constata-se o esperado: tanto no caso masculino quanto no feminino, a probabilidade de morte por idade se reduziu a cada ano. Isto significa que houve ganho de longevidade entre 2011 e 2016.

Figura 2 - Log da probabilidade de morte por idade da tábua do IBGE (feminino), de 2011 a 2016

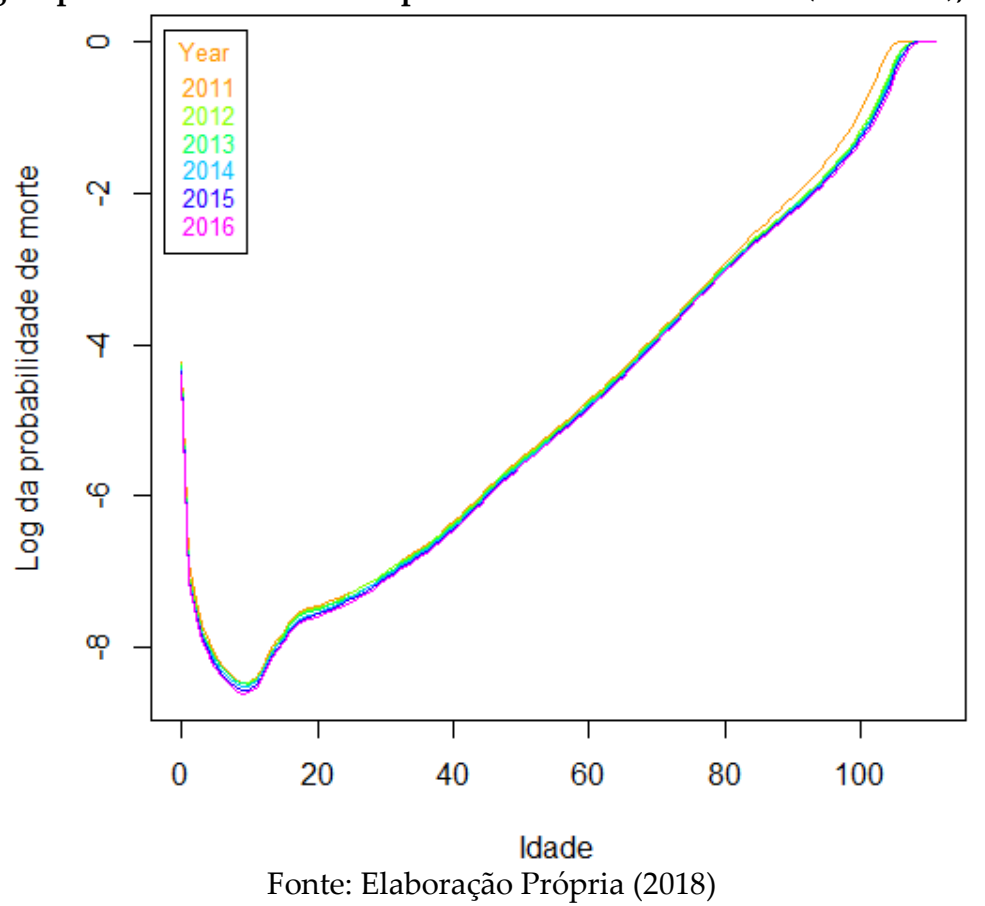

O passo seguinte é realizar o ajuste dos modelos de mortalidade e fazer predições. Para isso, utiliza-se de uma rotina de regressão que comporta seis variantes da classe de estruturas do modelo de Lee-Carter (por meio de método de Newton-Raphson iterativo). Estima-se, para ambas as tábuas IBGE (masculina e feminina), a Regressão Família Lee-Carter Iterativa, cujo modelo é $\ln \left(\mathrm{m}_{\mathrm{x}, \mathrm{t}}\right)=\mathrm{a}_{\mathrm{x}}+$ $\mathrm{b}_{\mathrm{x}} \times \mathrm{k}_{\mathrm{t}}$ com estrutura de erro Poisson, segundo a modelagem proposta por Renshaw e Haberman (2003). Tal modelo estima $\mathrm{a}_{\mathrm{x}}$ (para cada idade), $\mathrm{b}_{\mathrm{x}}$ (para cada idade) e $\mathrm{k}_{\mathrm{t}}$ (para cada ano).

Quanto à tábua masculina (Figura 3), obteve-se convergência em 6 iterações. O deviance médio basal é igual a 0,036 de um total de 0,078. 
Figura 3 - Regressão Lee-Carter Período-Idade para a tábua IBGE (masculino)
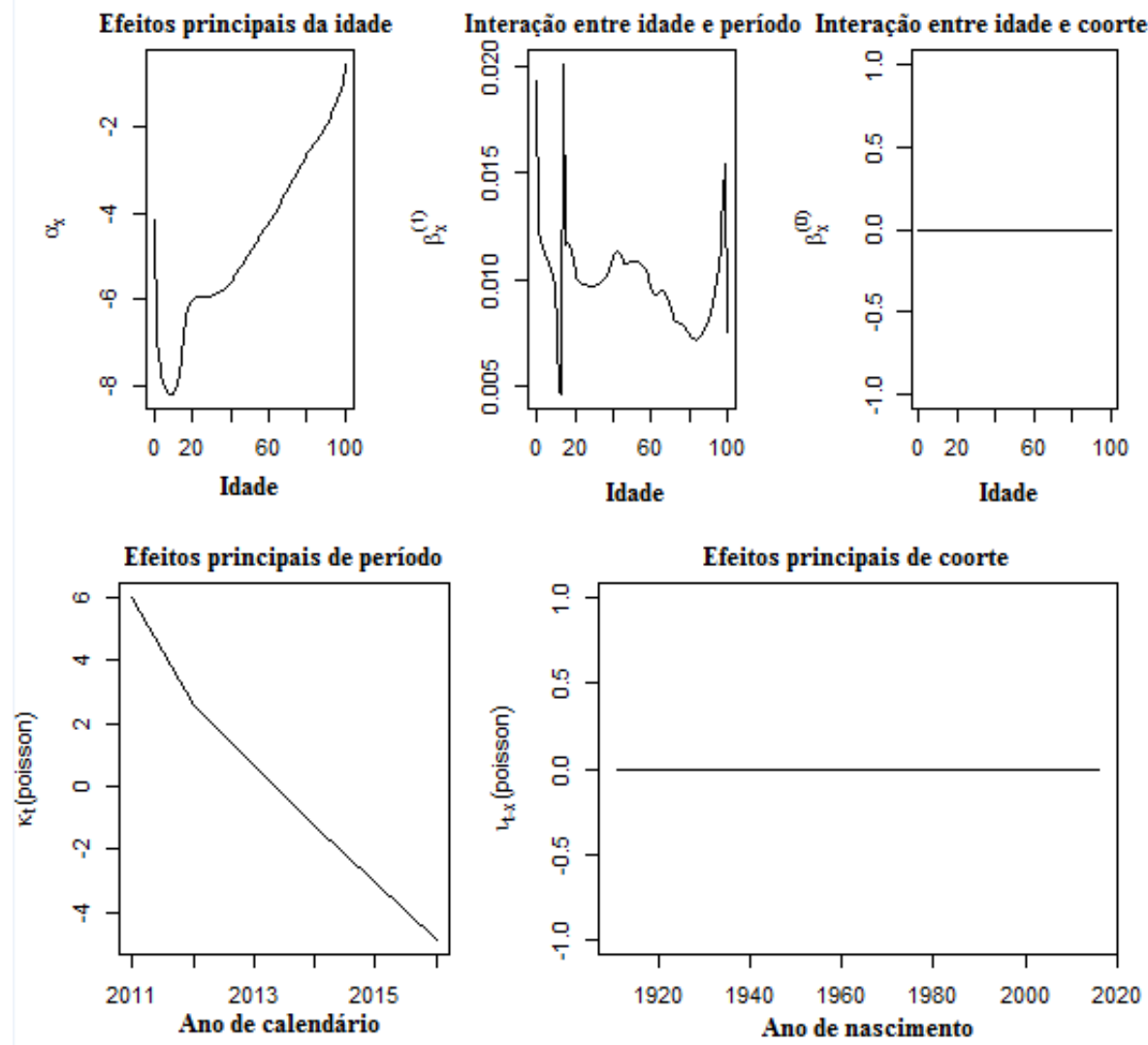

Fonte: Elaboração Própria (2018)

A Figura 3 apresenta alguns resultados da regressão, os efeitos principais de idade, período e coorte, além dos efeitos de interação de períodos e coorte, cada um associado a um parâmetro do Modelo de Lee-Carter Generalizado. Neste caso, não há efeito de coorte com o passar da idade e dos anos de nascimento, representando o caso particular Modelo de Lee-Carter Padrão. Além disso, verifica-se que $\mathrm{k}_{\mathrm{t}}$ suaviza $\mathrm{q}_{\mathrm{x}}$.

A Figura 4 apresenta previsão anual, de 2017 até 2026, do logaritmo das probabilidades de morte masculinas. 
Figura 4 - Logaritmo da probabilidade de morte prevista para a Tábua IBGE (masculino) entre 2017 e 2026

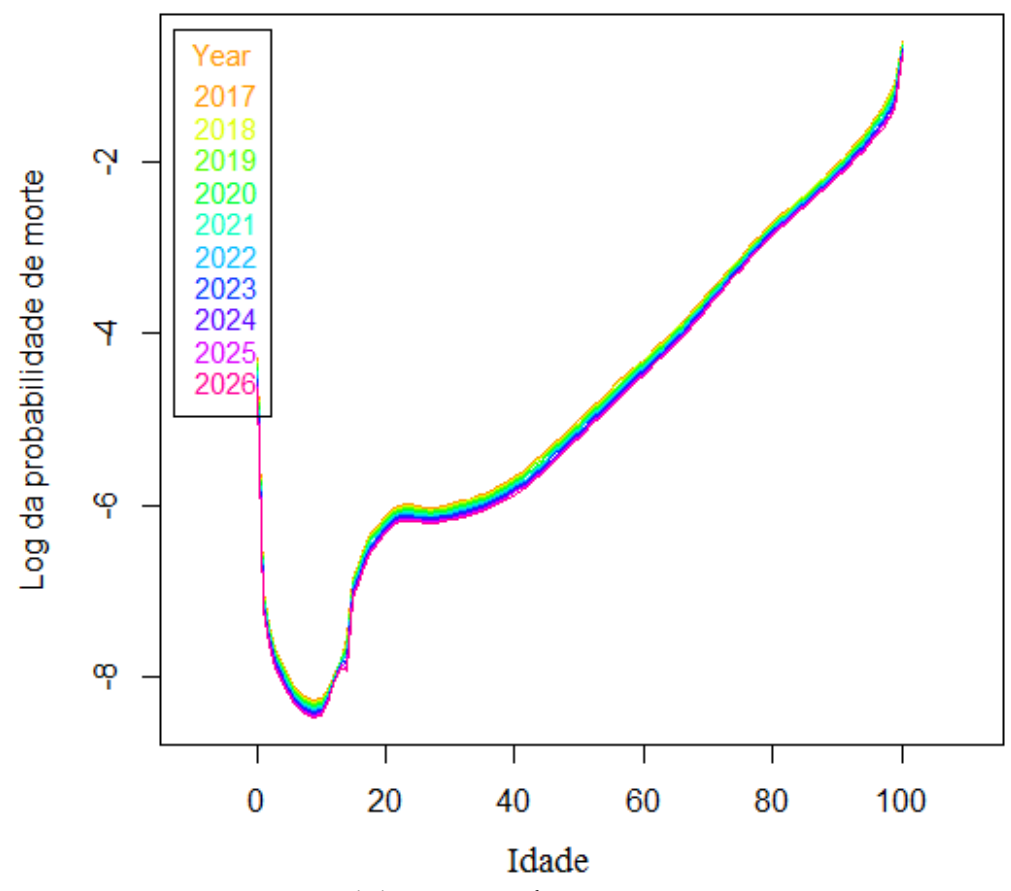

Fonte: Elaboração Própria (2018)

Quanto à tábua feminina, obteve-se convergência em 6 iterações. O deviance médio basal é igual a 0,14 de um total de 0,949. A Figura 5 apresenta alguns resultados dessa regressão, idem caso masculino.

Figura 5 - Regressão Lee-Carter Período-Idade para a tábua IBGE (feminino)
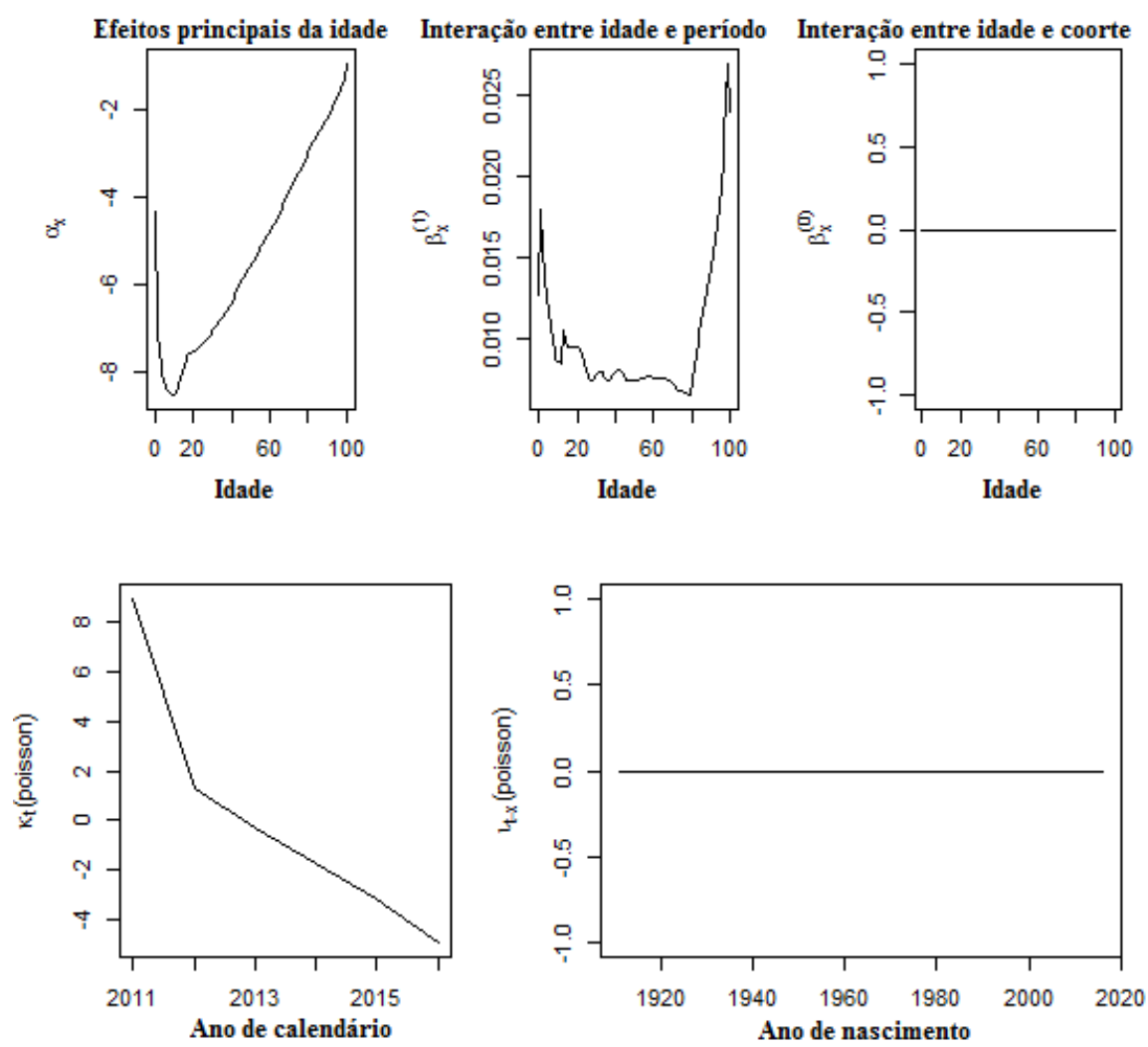

Fonte: Elaboração Própria (2018) 
A Figura 6 apresenta a previsão da tábua feminina, indicadora de ganho de longevidade prevista com o passar do tempo.

Figura 6 - Logaritmo da probabilidade de morte prevista para a Tábua IBGE (feminino) entre 2017 e 2026

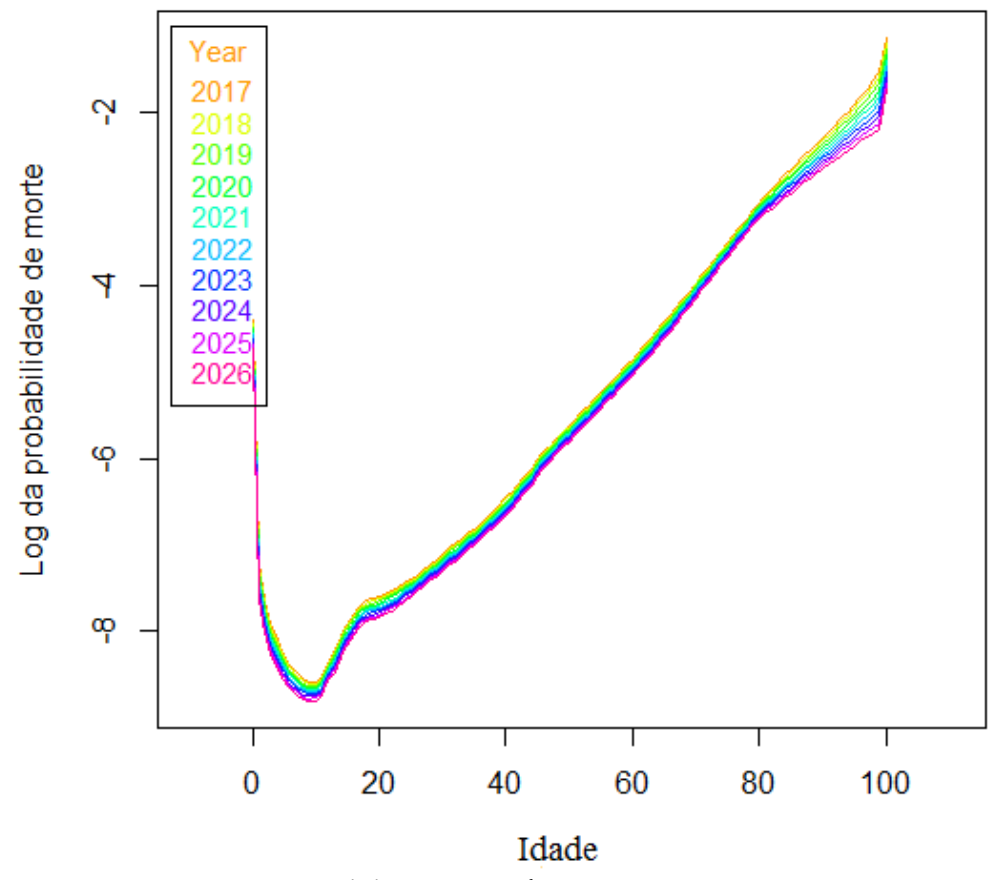

Fonte: Elaboração Própria (2018)

Comparando-se os resultados deste trabalho com os de Gallop (2008), tem-se que as mortalidades projetadas para o povo brasileiro são maiores que as de outros povos pertencentes a países desenvolvidos, tal qual a Inglaterra, conforme o esperado. Depois de preditas as mortalidades masculina e feminina brasileira e constatada a redução da mortalidade porvir para ambos os sexos, optou-se por estimar o tempo de vida média residual dos brasileiros do sexo masculino (Tabela 2) e feminino (Tabela 3), nas idades 0, 30 e 60 anos.

Consoante Ortega (1987), devido à mortalidade em relação ao primeiro ano (o número de mortes entre as idades 0 e 1 é muito alto), a expectativa de vida aumenta nas idades iniciais (acima de 1 ano de idade) e, em seguida, diminui continuamente até o final da vida. Em particular, se $\mathrm{x}=0$, obtém-se a expectativa de vida ao nascer $\left(\mathrm{e}_{\mathrm{x}}^{0}\right)$; além disso, $\left(\mathrm{x}+\mathrm{e}_{\mathrm{x}}^{0}\right)$ denota o número total de anos que em média vive uma pessoa de idade $\mathrm{x}$. 
Tabela 2 - Projeções do tempo de vida média residual dos brasileiros, sexo masculino

\begin{tabular}{|c|c|c|c|c|c|c|c|}
\hline \multirow{2}{*}{ Ano } & \multicolumn{2}{|c|}{0 anos } & \multicolumn{2}{|c|}{30 anos } & \multicolumn{3}{|c|}{60 anos } \\
\hline & Absoluto & Relativo & Absoluto & Relativo & Absolut & & Relativo \\
\hline 2017 & 72,7618 & - & 45,81 & - & & & - \\
\hline 2018 & 73,0841 & $0,4430 \%$ & 46,06 & $0,5428 \%$ & 20 , & & $0,8158 \%$ \\
\hline 2019 & 73,4038 & $0,4374 \%$ & 46,31 & $0,5388 \%$ & & & 0,8111\% \\
\hline 2020 & 73,7210 & $0,4321 \%$ & 46,55 & $0,5344 \%$ & & & $0,8069 \%$ \\
\hline 2021 & 74,0359 & $0,4272 \%$ & 46,80 & $0,5305 \%$ & & & $0,8028 \%$ \\
\hline 2022 & 74,3484 & $0,4221 \%$ & 47,05 & $0,5264 \%$ & & & $0,7983 \%$ \\
\hline 2023 & 74,6586 & $0,4172 \%$ & 47,29 & $0,5224 \%$ & & & $0,7938 \%$ \\
\hline \multirow[t]{2}{*}{ Ano } & \multicolumn{2}{|c|}{0 anos } & \multicolumn{2}{|c|}{30 anos } & \multicolumn{3}{|c|}{60 anos } \\
\hline & Absoluto & Relativo & Absoluto & Relativo & Absoluto & & \\
\hline 2024 & 74,9666 & $0,4125 \%$ & 47,5428 & $0,5186 \%$ & 21,9739 & 0,7 & \\
\hline 2025 & 75,2723 & $0,4078 \%$ & 47,7876 & $0,5149 \%$ & 22,1466 & 0,7 & $\%$ \\
\hline 2026 & 75,5760 & $0,4035 \%$ & 48,0318 & $0,5110 \%$ & 22,3197 & 0,7 & \\
\hline
\end{tabular}

Por meio da visualização da Tabela 2, é possível perceber que o tempo restante de vida aumentará para todas as idades; quanto maior a idade, maiores os aumentos, conforme as colunas de ganho relativo (Relativo). Esse efeito decorre do processo de inversão do ganho de sobrevida, em que os grupos etários de maior idade passam a deter maior ganho em sobrevida em um contexto que há conformação da queda da mortalidade nas primeiras idades (Turra, 2012). A mortalidade nas primeiras idades responde, em geral, pelos maiores ganhos na expectativa de vida, no entanto, em sociedades onde há uma consolidação da queda da mortalidade infantil e na infância, onde também há um processo de envelhecimento desencadeado, os grupos etários mais velhos respondem pelos maiores ganhos em sobrevida (Tuljapurkar, \& Boe, 1998).

Tabela 3 - Projeções do tempo de vida média residual dos brasileiros, sexo feminino

\begin{tabular}{ccccccc}
\hline \multirow{2}{*}{ Ano } & \multicolumn{2}{c}{0 anos } & \multicolumn{2}{c}{30 anos } & \multicolumn{2}{c}{60 anos } \\
\cline { 2 - 7 } 2017 & Absoluto & Relativo & Absoluto & Relativo & Absoluto & Relativo \\
\hline 2018 & 79,8490 & - & 51,6103 & - & 24,4673 & - \\
2019 & 80,1994 & $0,4388 \%$ & 51,9128 & $0,5861 \%$ & 24,7338 & $1,0891 \%$ \\
2020 & 80,5553 & $0,4437 \%$ & 52,2223 & $0,5961 \% ?$ & 25,0081 & $1,1091 \%$ \\
2021 & 80,9173 & $0,4494 \%$ & 52,5393 & $0,6071 \%$ & 25,2909 & $1,1308 \%$ \\
2022 & 81,2861 & $0,4558 \%$ & 52,8646 & $0,6191 \%$ & 25,5828 & $1,1543 \%$ \\
2023 & 81,6625 & $0,4630 \%$ & 53,1989 & $0,6323 \%$ & 25,8847 & $1,1799 \%$ \\
2024 & 82,0473 & $0,4712 \%$ & 53,5429 & $0,6467 \%$ & 26,1973 & $1,2075 \%$ \\
2025 & 82,4414 & $0,4804 \%$ & 53,8975 & $0,6624 \%$ & 26,5214 & $1,2374 \%$ \\
2026 & 82,8458 & $0,4906 \%$ & 54,2638 & $0,6795 \%$ & 26,8582 & $1,2698 \%$ \\
\hline
\end{tabular}

Fonte: Elaboração Própria (2018)

A Tabela 3 exibe a projeção do tempo de vida média residual do grupo feminino com idades 0, 30 e 60 anos. Por meio de sua visualização, é possível perceber que o tempo restante de vida aumentará para todas as idades; os maiores aumentos ocorrerão para os idosos, conforme ganho relativo. 
É possível perceber que o tempo de vida média residual, para qualquer idade, é sempre maior para o grupo feminino, conforme informação absoluta; no entanto, o aumento da expectativa de vida, qual seja, o ganho de longevidade, do grupo masculino é maior, conforme informação relativa.

Assim, o modelo LC previu o comportamento da mortalidade do brasileiro por meio da série histórica da Tábua IBGE. Os resultados seguem a tendência apontada por Li, Lee e Gerland (2013), em que se detectou a presença de rotação, isto é, maior redução de mortalidade entre os mais velhos que os mais novos.

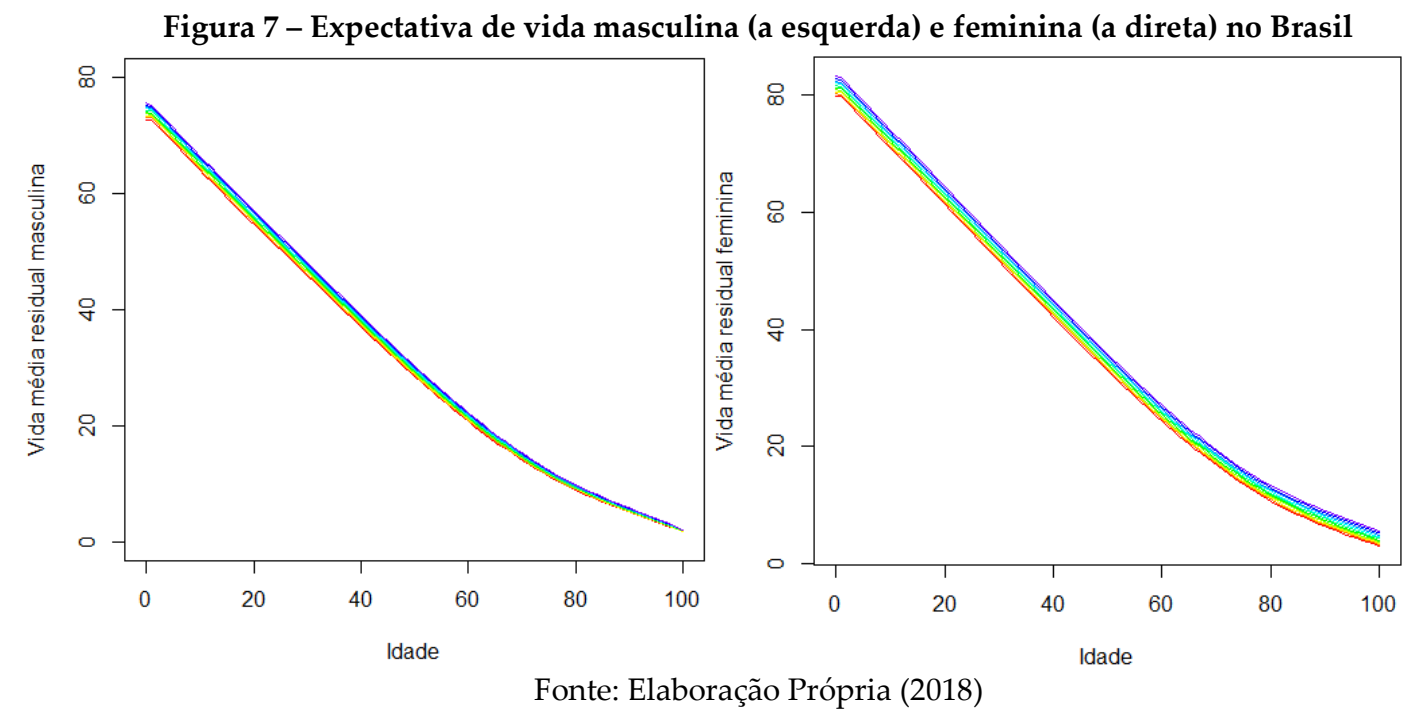

A Figura 7 exibe, ainda, as linhas de expectativa de vida prevista para todas as idades face a face o ano (2017 a 2026), por sexo. Trata-se, portanto, de uma versão gráfica e mais completa das informações apresentadas nas Tabelas 2 e 3.

\subsection{Efeitos das Mortalidades Estimadas sobre o Cálculo Atuarial}

Conforme ASB (2014), a mortalidade é uma premissa estimada para realização de cálculos atuariais. Nesta subseção se apresentam os efeitos das diferentes estimativas de mortalidade sobre tais cálculos, viabilizados a partir de uma massa hipotética de segurados, composta por 850 ativos, 120 aposentados e 30 pensionistas. Aqui, utilizou-se da série de tábuas IBGE ambos (não segregadas por sexo), em conformidade com a prática atuarial em RPPS. Na data-base 31/12/2016, a idade média de entrada no mercado de trabalho é de 28,34 anos e a idade média de aposentadoria é de 58,54 anos. Foram supostas, ainda, as seguintes hipóteses:

- Idade mínima de Entrada no Mercado de Trabalho: 18 anos.

- Taxa real de juros anual: $6 \%$.

- Crescimento salarial anual: $1 \%$.

- Crescimento de benefícios: 0 .

- Probabilidade de ser casado: $85 \%$.

- Idade de aposentadoria compulsória: 70 anos.

- Tábua de entrada em invalidez: Álvaro Vindas.

- Tábua de mortalidade de inválidos: AT 49 (para ambos os sexos).

- Custo normal sob a forma: $12 \times a_{x}^{12}+a_{x}$ ( $a_{x}$ representa anuidades ou rendas vitalícias). 
O Quadro 1 apresenta os benefícios considerados nos cálculos, bem como o regime de financiamento (e método de custeio, em caso de capitalização).

Quadro 1 - Benefícios e respectivos regimes financeiros

\begin{tabular}{|l|c|c|}
\hline Benefício & Regime Financeiro & Método de financiamento \\
\hline Aposentadorias com reversão ao dependente & Capitalização & PUC \\
\hline Invalidez com reversão ao dependente & Capital de Cobertura & - \\
\hline Pensão de participantes em atividade & Capital de Cobertura & - \\
\hline
\end{tabular}

Fonte: Elaboração Própria (2018)

A Tabela 4 apresenta a variação percentual do custo normal e reserva matemática quando se consideram as diferentes mortalidades, em relação à tábua IBGE 2016 observada: i) IBGE 2014 observada; ii) IBGE 2016 estimada pela escala AA; iii) IBGE 2016 estimada por LC; iv) IBGE 2016 estimada por MQM. É possível verificar que, para os dados simulados, as variações percentuais de CN e RM são pequenas (absolutamente) e pouco variam (relativamente), quando comparadas ao cenário-referência intitulado "Observada 2016", a tábua considerada mais adequada para cálculo referente ao ano-base 2016.

Constata-se que "Observada 2014" é a tábua efetivamente usada em cálculos atuariais para o ano-base 2016, dado que é comum o uso, à época, da tábua mais recentemente disponibilizada pelo IBGE, o que pressupõe uma defasagem de aproximadamente dois anos; "AA 2016", "LC 2016" e "MQM 2016" apresentam as estimativas de mortalidades calculadas neste trabalho, por meio dos modelos apresentados nas subseções 3.3.1, 3.3.2 e 3.3.3, respectivamente.

Tabela 4 - Análise de Sensibilidade

\begin{tabular}{lcccc}
\hline Tábua & $\mathrm{CN}(\%)$ & $\mathrm{CN}(\Delta \%)$ & $\mathrm{RM}(\mathrm{R} \$)$ & $\mathrm{RM}(\Delta \%)$ \\
\hline Observada 2014 & 37,77 & $-0,18$ & $189.985 .785,27$ & $-0,83$ \\
Observada 2016 & 37,84 & - & $191.571 .267,28$ & - \\
AA 2016 & 37,81 & $-0,08$ & $191.181 .162,09$ & $-0,20$ \\
LC 2016 & 37,81 & $-0,08$ & $189.627 .456,77$ & $-1,01$ \\
MQM 2016 & 38,09 & 0,66 & $193.745 .494,45$ & 1,13 \\
\hline
\end{tabular}

Fonte: Elaboração Própria (2018)

Para melhor compreender as nuances proporcionadas pela mortalidade de cada tábua, optouse pelo detalhamento das contas que compõem CN e RM.

Tabela 5 - Composição do Custo Normal

\begin{tabular}{lccccc}
\hline & Obs 2014 & Obs 2016 & AA 2016 & LC 2016 & MQM 2016 \\
\hline Aposentadorias com reversão ao dependente (+) & $26,88 \%$ & $27,11 \%$ & $27,06 \%$ & $26,81 \%$ & $27,35 \%$ \\
Invalidez com reversão ao dependente (+) & $3,53 \%$ & $3,54 \%$ & $3,54 \%$ & $3,53 \%$ & $3,55 \%$ \\
Pensão de participantes em atividade (=) & $5,37 \%$ & $5,19 \%$ & $5,22 \%$ & $5,47 \%$ & $5,19 \%$ \\
\hline Custo Normal Anual Líquido (+) & $35,77 \%$ & $35,84 \%$ & $35,81 \%$ & $35,81 \%$ & $36,09 \%$ \\
Administração do Plano (=) & $2,00 \%$ & $2,00 \%$ & $2,00 \%$ & $2,00 \%$ & $2,00 \%$ \\
\hline Custo Normal Anual Total & $37,77 \%$ & $37,84 \%$ & $37,81 \%$ & $37,81 \%$ & $38,09 \%$ \\
\hline
\end{tabular}

Fonte: Elaboração Própria (2018)

Assim, a Tabela 5 apresenta a composição do CN para cada tábua considerada nos cálculos. Indica-se que, em relação a Observado 2016, as tábuas Observado 2014, AA 2016, e LC 2016: subestimam as alíquotas referentes ao CN calculado, em virtude da subestimação da alíquota de benefícios de aposentadorias, que possuem maior peso em sua composição; subestimam ou igualam as alíquotas referentes aos benefícios de invalidez; superestimam as alíquotas referentes aos benefícios 
de pensão. A tábua MQM, por sua vez, superestima a parcela referente à aposentadoria programada e de invalidez e iguala a de pensão, de modo que se superestima a alíquota de CN.

A Tabela 6 apresenta a composição da RM para cada tábua considerada nos cálculos. Indicase que, em relação a Observado 2016, as tábuas Observado 2014, AA 2016, e LC 2016: subestimam RM, em virtude da subestimação da Reserva Matemática de Benefícios Concedidos (RMBC) e Reserva Matemática de Benefícios a Conceder (RMBaC). A tábua MQM 2016, ao contrário, superestima RM, em decorrência da superestimação de todos os itens de RMBC e RMBaC.

Tabela 6 - Composição da Reserva Matemática

\begin{tabular}{|c|c|c|c|c|c|c|}
\hline \multicolumn{2}{|c|}{ Discriminação } & Obs 2014 & Obs 2016 & AA 2016 & LC 2016 & MQM 2016 \\
\hline \multirow{4}{*}{$\mathrm{BC}$} & (-) VPBF (apos.) & $46.585 .822,48$ & $46.905 .850,20$ & 46.811.401,22 & $46.574 .017,05$ & $47.648 .755,13$ \\
\hline & (+) VPCF (apos.) & $279.998,00$ & $281.967,96$ & $281.431,78$ & $279.873,96$ & $285.578,61$ \\
\hline & (-) VPBF (pens.) & $8.359 .265,84$ & $8.447 .124,07$ & $8.418 .963,82$ & $8.343 .663,76$ & $8.698 .102,75$ \\
\hline & (+) VPCF (pens.) & $50.805,78$ & $51.976,03$ & $51.433,28$ & $50.848,00$ & $58.947,92$ \\
\hline \multicolumn{2}{|c|}{$(=)$ RMBC } & $54.614 .284,53$ & $55.019 .030,29$ & $54.897 .499,99$ & $54.586 .958,85$ & $56.002 .331,36$ \\
\hline \multirow{2}{*}{$\mathrm{BaC}$} & (-) VPBF & $213.744 .474,85$ & $215.608 .795,25$ & $215.184 .729,63$ & $213.221 .838,82$ & $217.489 .204,89$ \\
\hline & $(+) \mathrm{VPCF}$ & $78.372 .974,11$ & $79.056 .558,26$ & $78.901 .067,53$ & $78.181 .340,90$ & $79.746 .041,79$ \\
\hline \multicolumn{2}{|c|}{$(=) \mathrm{RMBaC}$} & $135.371 .500,74$ & $136.552 .236,99$ & $136.283 .662,10$ & $135.040 .497,92$ & $137.743 .163,09$ \\
\hline \multicolumn{2}{|c|}{$\mathrm{RM}(\mathrm{RMBC}+\mathrm{RMBaC})$} & $189.985 .785,27$ & $191.571 .267,28$ & 191.181.162,09 & $189.627 .456,77$ & $193.745 .494,45$ \\
\hline
\end{tabular}

Fonte: Elaboração Própria (2018)

Segundo Winklevoss (1993), o tamanho relativo do passivo atuarial auferido pelo PUC é menor que àquele auferido pelo IEN. Neste sentido, as diferenças proporcionadas pela mudança de tábua podem ser ainda maiores que as apresentadas aqui, de acordo com o método de custeio adotado.

Observa-se, visualizando-se a Tabela 7, que as mortalidades utilizadas para comparar as variações de CN e RM são aderentes em relação àquela denominada "cenário-referência", conforme indica o teste KS. Como os valores p são superiores ao nível de significância de 5\%, não existem evidências para rejeitar a hipótese nula de que as distribuições de Observada 2014, AA 2016, LC 2016 e MQM 2016 se diferem da distribuição de Observada 2016.

Tabela 7 - Teste de aderência KS e MAPE

\begin{tabular}{lccc}
\hline Mortalidades comparadas & Estatística & Valor p & MAPE \\
\hline Observada 2016, Observada 2014 & 0,07 & 0,9671 & $4,34 \%$ \\
Observada 2016, AA 2016 & 0,05 & 0,9996 & $1,36 \%$ \\
Observada 2016, LC 2016 & 0,08 & 0,9062 & $5,48 \%$ \\
Observada 2016, MQM 2016 & 0,11 & 0,5816 & $22,17 \%$ \\
\hline
\end{tabular}

Fonte: Elaboração Própria (2018)

A Tabela 7 também apresentou valores da MAPE. Como quanto menor o MAPE, melhor o ajuste, a escala AA, para este caso, apresentou melhores estimativas.

\section{CONCLUSÃO}

A limitação inicial apresentada pelo uso de tábua abreviada - os modelos de mortalidade que não incluem todas as faixas etárias produzem projeções questionáveis - foi solucionada com o uso do método de Castro (2018) para sua extrapolação. Neste sentido, destaca-se a importância dos métodos de estimação e análise da mortalidade para as idades mais avançadas. 
Utilizando-se de uma variação do Método de Lee-Carter - que inclui fatores pertinentes à coorte, período e idade (Booth, \& Tickle, 2008) -, o mais citado dentre os métodos de previsão de mortalidade (Santos, 2007), e a partir dos dados contidos nas tábuas de vida fornecidas pelo IBGE, de 2011 a 2016, foi possível projetar e caracterizar as taxas de mortalidade do brasileiro, por sexo, e seu tempo de vida média residual, com destaque para a ocorrência da rotação.

Para verificação do efeito da mortalidade, consoante seu método de previsão, sobre o cálculo atuarial, utilizaram-se da escala AA, do modelo de Lee-Carter estendido e do modelo quadrático múltiplo. Conforme MAPE, a escala AA foi considerada a mais adequada para os dados aqui utilizados. Isso significa que a RM calculada em 2016, para o RPPS (de pequeno porte) fictício investigado, a tábua IBGE 2014 poderia ter sido substituída pela tábua AA afim de obter resultados mais fidedignos.

Dentre as limitações do presente trabalho, bem como sugestões para futuras pesquisas, apontam-se: o uso de apenas um método de custeio, qual seja, PUC, é limitado, sugerindo-se o uso de outros métodos, tal qual o IEN, para verificação mais ampla do impacto das variações das tábuas de vida sobre as reservas matemáticas; o uso de uma série histórica de apenas 6 anos (IBGE 2011-2016) pode ser superado pelo uso de todas as tábuas disponibilizadas pelo IBGE, bem como de outras tábuas abreviadas, para extrapolação da mortalidade; indica-se, ainda a utilização de outros métodos de extrapolação, de previsão, bem como a aplicação das estimativas de mortalidade a dados reais, qual seja, nos segmentos de seguro, previdência ou saúde.

Apesar do interesse, no presente estudo, em mensurar o impacto das diferentes estimativas de mortalidade sobre o cálculo atuarial, seus efeitos ecoam, na verdade, sobre diversas áreas: sob a perspectiva demográfica, a mortalidade se constitui num dos três componentes (junto com a fecundidade e migração) que determinam o ritmo do crescimento populacional; sob o olhar das políticas públicas, em que a redução da mortalidade em alguma ou em todas as faixas etárias devem nortear a oferta de serviços públicos; sob a ótica previdenciária, define o tipo de relação entre ativos e inativos. Assim, de forma menos restrita, é possível compreender a importância de estudos relativos à mortalidade e suas inúmeras aplicações.

\section{REFERÊNCIAS}

Azevedo, Felipe I. X. Modelo Quadrático Múltiplo (MQM) para estimativas de mortalidade: um exercício metodológico para o Brasil (1998-2016) e Chile (1992-2008). (Anais). VIII Congresso de la Asociación Lattinoamericana de Población. Puebla, México, out. 2018.

Beltrão, Kaizo I., \& Sugahara, Sonoê. (2002). Tábua de mortalidade para os funcionários públicos civis federais do poder executivo por sexo e escolaridade: comparação com tábuas do mercado. Rio de Janeiro: Escola Nacional de Ciências Estatísticas, 2002. 43p. - (Textos para discussão. Escola Nacional de Ciências Estatísticas, ISSN 1677-7093; n. 3). Disponível em: <https://biblioteca.ibge.gov.br/visualizacao/livros/liv1419.pdf>

Beltrão, Kaizo I., \& Sugahara, Sonoê. (2017). Mortalidade dos funcionários públicos civis do Executivo por sexo e escolaridade - 1993/2014. R. Cont. Fin. - USP, São Paulo, 28(75), 445-464, set./dez. 2017. Disponível em: <http://www.revistas.usp.br/rcf/article/view/138289/133739>

Booth, H., \& Tickle, L. (2008). Mortality modelling and forecasting: a review of methods. Annals of Actuarial Science, The Faculty of Actuaries and Institute of Actuaries, 3(9). Disponível em: $<$ http://www.ressourcesactuarieles.net/EXT/ISFA/1226.nsf/0/8e4362531df5fbcdc125785d005906d6/\$FILE/Booth_Tickle_2008.pdf> 
Bowers, N. L., Gerber, H. U., Hickman, J. C., Jones, D.A. and Nesbitt, C. J. (1997). Actuarial Mathematics, 2nd edition. Itasca: Society of Actuaries.

Brasil. (2008). Portaria no 403, de 10 de dezembro de 2008. Dispõe sobre as normas aplicáveis às avaliações e reavaliações atuariais dos Regimes Próprios de Previdência Social - RPPS da união, dos estados, do distrito federal e dos municípios, define parâmetros para a segregação da massa e dá outras providências. Lex, Brasília. Disponível em: <http://sislex.previdencia.gov.br/paginas/66/MPS/2008/403_1.htm>

Bravo, J. M. V. (2007). Tábuas de mortalidade contemporâneas e prospectivas: modelos estocásticos, aplicações actuariais e cobertura do risco de longevidade. 566 p. (Tese de Doutorado em Economia) | Universidade de Évora, Évora. Disponível em: <http://home.uevora.pt/ braumann/project_papers/JMB07-PhDThesis-JorgeMiguelBravo.pdf $>$

Butt, Z., Haberman, S., \& Shang, H. L. (2014). The ilc Package in R: Generalised Lee-Carter models using iterative fitting algorithms. Tese I University of London. Disponível em: $<$ https://www.researchgate.net/publication/271013835_The_ilc_Package_in_R_Generalised_Lee-Carter_models_using_iterative_fitting_algorithms/download>

Camarano, Ana A., \& Kanso, Solange. (2009). Perspectivas de crescimento para a população brasileira: velhos e novos resultados. Texto para Discussão № 1426. Instituto de Pesquisa Econômica Aplicada, Rio de Janeiro, out. 2009. Disponível em: <http://www.ipea.gov.br/portal/images/stories/PDFs/TDs/td_1426.pdf>

Castro, L. G. (2018) Nota técnica sobre a metodologia adotada pelo ministério da previdência social na extrapolação das tábuas de mortalidade IBGE para as idades acima de 80 anos. IBGE, 1-47. Disponível em: < http://sa.previdencia.gov.br/site/2015/06/NOTA-TECNICA-ATUARIAL-EXTRAPOLACAODA-TABUA-IBGE-MPS.pdf >

Colatto, Marcos; Dias, Cícero Rafael Barros. Método de custeio dos cálculos atuariais: uma análise nos Regimes Próprios de Previdência Social. In: BRASIL. Regimes Previdenciários e Situação Atuarial. Brasília: Secretaria da Previdência, 2018. Cap. 14. p. 340-365.

Colosimo, Enrico Antônio, \& Giolo, Suely Ruiz. Análise de Sobrevivência Aplicada. (2006). São Paulo: Blucher.

Correia, Cristiane Silva. Premissas atuariais em planos previdenciários: uma visão atuarial-demográfica. Curitiba: Appris, 2018.

Deprez, P., Shevchenko, P. V., Wüthrich, M. V. (2017). Machine learning techniques for mortality modeling. European Actuarial Journal 7/2, 337-352.

Dickson, D. C. M. (2006). Premiums and reserves for life insurance products. Australian Actuarial Journal 12, 259-79.

Elandt-Johnson, Regina C., \& Johnson, Normanl. (1980). Survival Models and Data Analysis. New York: John Wiley \& Sons, 457p.

Gallop, A. (2008). Mortality projections in the United Kingdom. Living to 100 and Beyond Symposium. Disponível em: <http://citeseerx.ist.psu.edu /viewdoc/download?doi=10.1.1.498.1133\&rep $=$ rep1\&type $=$ pdf $>$

Gonzaga, Marcos R., \& Schmertmann, Carl P. (2016). Estimativa de taxas de mortalidade por idade e sexo para pequenas áreas com regressão de TOPALS: uma aplicação para o Brasil em 2010. 
Rev. Bras. Estud. Popul. [online]. 2016, 33(3), 629-652. ISSN 0102-3098. Disponível em: $<$ http://dx.doi.org/10.20947/s0102-30982016c0009>.

Huang, F., \& Browne, B. (2017). Mortality forecasting using a modified continuous mortality investigation mortality projections model for china i: methodology and country-level results. Annals of Actuarial Science, The Faculty of Actuaries and Institute of Actuaries, 11. Disponível em: http://gen.lib.rus.ec/scimag/index.php?s=10.1017/s1748499516000142.

Judson, D. H., \& Popoff, Carole L. (2004). Selected General Methods. In: Siegel, Jacob S., Swanson, David A. (eds), The methods and materials of demography. Ed 2. New York: Elsevier Academic Press. 835 p.

Khoury, Felipe Kaiuca Castelo Branco. (2011). Minimização de custos de produção via programação inteira mista: estudo de caso de planejamento de produção de luminárias. 2011. $114 \mathrm{f}$. Dissertação (Mestrado) - Curso de Engenharia de Produção, Engenharia Industrial, Pontifícia Universidade Católica do Rio de Janeiro, Rio de Janeiro. Disponível em: https://www.maxwell.vrac.pucrio.br/18828/18828_1.PDF

Lee, R. D., \& Carter, L. (1992). Modeling and forecasting the time series of us mortality. Journal of the American Statistical Association, 87(419), 659- 671. Disponível em: <http://pagesperso.univbrest.fr/ ailliot/doc_cours/M1EURIA/regression/leecarter.pdf >

Li, N., Lee, R., \& Gerland, P. (2013). Extending the lee-carter method to model the rotation of age patterns of mortality decline for long-term projections. Demography, Springer US, 50(12). Disponível em: http://gen.lib.rus.ec/scimag/index.php?s=10.1007/ s13524-013-0232-2.

Miranda, Gabriella M. D., Mendes, Antonio C. G., \& Silva, Ana L. A. (2016). O envelhecimento populacional brasileiro: desafios e consequências sociais atuais e futuras. Rev. Bras. Geriatr. Gerontol., Rio de Janeiro, 19(3), 507-519. Disponível em: <http://www.scielo.br/scielo.php?pid=S180998232016000300507\&script=sci_arttext\&tlng=pt >

Monteiro, J. R., \& Leão, L. C. S. (2012). Risco Atuarial no contexto da Supervisão Baseada em Riscos para Fundos de Pensão: um estudo sobre Tábuas de Mortalidade e Taxas de Juros. 59 p. (TCC de Graduação do Curso de Ciências Atuariais I Universidade Estadual do Rio de Janeiro, Rio de Janeiro.

Myrrha, Luana J. D., Siviero, Pamila C. L., Wajnman, Simone, \& Turra, Cássio M. (2014). O uso das taxas de crescimento por idade para identificação das principais etapas da transição demográfica no Brasil. R. Bras. Est. Pop., Rio de Janeiro, 31(2), p. 259-275, jul./dez. Disponível em: $<$ http://www.scielo.br/pdf/rbepop/v31n2/a02v31n2.pdf>

Namboodiri, K. (1991): Demographic Analysis: A Stochastic Approach. Academic Press, Inc.

Nunes, Felipe C., \& Carvalho, J. M. B. L. M. (2008) Tábuas geracionais: uma aplicação em planos de benefícios de entidades fechadas de previdência complementar. Associação Brasileira das Entidades Fechadas de Previdência Complementar - ABRAPP, 29ํㅡㄹ Congresso Brasileiro dos Fundos de Pensão. Discutindo conceitos: realidade de um novo tempo. Rio de Janeiro, 30 p. Disponível em: <https://www.artigos.com/artigos-academicos/8290-tabuas-geracionais-uma-aplicacao-emplanos-de-beneficios-de-entidades-fechadas-de-previdencia-complementar>

Oliveira, M., Frischtak, R., Ramirez, M., Beltrão, K., \& Pinheiro, S. (2012). Tábuas biométricas de mortalidade e sobrevivência: experiência do mercado segurador brasileiro 2010 / Mário de Oliveira ... / et al/. -- Rio Janeiro: Funenseg. 112p. Disponível em: <http://labma.ufrj.br/site/static/arquivos/Livro_Tabuas_Portugues.pdf> 
Ortega, A. Tablas de Mortalidad. (1987). San José: Centro Latinoamericano de Demografia.

Pinheiro, Ricardo Pena. A demografia dos fundos de pensão. / Ricardo Pena Pinheiro. - Brasília: Ministério da Previdência Social. Secretaria de Políticas de Previdência Social. Coleção Previdência Social. Série estudos; v. 24, 2007. 292 p.

Renshaw, A. E., \& Haberman, S. (2003). Lee-carter mortality forecasting with age-specific enhancement. Insurance: Mathematics and Economics, 33(2), 255-272.

Ribeiro, M. M., Turra, C. M., \& Pinto, C. C. X. (2016). Estimativas de mortalidade adulta por níveis de escolaridade no estado São Paulo em 2010. (Anais). VII Congreso de la Asociación LatinoAmericana de Población e XX Encontro Nacional de Estudos Populacionais, realizado em Foz do Iguaçu/PR - Brasil, out. Disponível em: <http://abep.org.br/xxencontro/files/paper/838-717.pdf>

Richman, R. (2018). AI in actuarial science. SSRN Manuscript ID 3218082, Version of August 20, 2018.

Rodrigues, José Angelo. Gestão de Risco Atuarial. São Paulo: Saraiva, 2008.

Sakr, Sherif \& El Shawi, Radwa \& Ahmed, Amjad \& Qureshi, Waqas \& Brawner, Clinton \& Keteyian, Steven \& Blaha, Michael \& Al-Mallah, Mouaz. (2017). Comparison of machine learning techniques to predict all-cause mortality using fitness data: The Henry Ford exercIse testing (FIT) project. BMC Medical Informatics and Decision Making (2017) 17:174. DOI 10.1186/s12911-0170566-6

Santos, R. R. (2007). Técnicas de Modelagem do Improvement para Construção de Tábuas Geracionais. 78 p. Dissertação (Mestrado) I Pós-Graduação em Ciências Atuariais, Pontifícia Universidade Católica do Rio de Janeiro, Rio de Janeiro. Disponível em: <http://livros01.livrosgratis.com.br/cp081644.pdf>

Silva, Fabiana L. (2010). Impacto do risco de longevidade em planos de previdência complementar. (Tese de Doutorado). Universidade de São Paulo, Programa de Pós-Graduação em Ciências Contábeis. São Paulo, 208 p. Disponível em: <http://www.teses.usp.br/teses/disponiveis/12/12136/tde29112010-182036/pt-br.php>

Souza, Felipe H. de. (2014). Padrão da mortalidade brasileira: estimativas a partir do nível municipal / Felipe Henrique de Souza. (Dissertação) - Natal, 2014. 105 f.: il. Disponível em: <http://arquivos.info.ufrn.br/arquivos/20150921072eea2422919260cd4cca8dc/Padro_da_Mortalidade_Brasilleira_Estimativas_a_partir_do_Nvel_Municipal.pdf>

Tuljapurkar, Shripad, \& Boe, Carl. (1998) Mortality Change and Forecasting. How Much and How Little Do We Know? North American Actuarial Journal, 2(4), 1347, DOI: 10.1080/10920277.1998.10595752.

Turra, Cássio M. (2012). Os limites do corpo: a longevidade em uma perspectiva demográfica. Rev. UFMG, Belo Horizonte, 19(1 e 2), 156-181, jan./dez. Disponível em: <https://www.ufmg.br/revistaufmg/pdf/REVISTA_19_web_156-181.pdf>

Wilbert, M. D., Lima, D. V., \& Gomes, M. M. F. (2013). O Impacto da Utilização de Diferentes Tábuas de Mortalidade nas Estimativas de Pagamento de Benefícios no RGPS. R. Bras. Risco e Seg., Rio de Janeiro, 8(16), 19-40, maio/out. Disponível em: <http://www.rbrs.com.br/arquivos/rbrs_16_2.pdf>

Winklevoss, H. E. Pension mathematics with numerical illustrations. 2 . ed. Philadelphia: Pension Research Council, 1993.309 p. 